\title{
Common fixed point theorem for two pairs of non-self-mappings satisfying generalized Ćirić type contraction condition in cone metric spaces
}

\author{
Xianjiu Huang ${ }^{1}$, Jing Luo', Chuanxi Zhu ${ }^{1 *}$ and Xi Wen²
}

"Correspondence:

chuanxizhu@126.com

${ }^{1}$ Department of Mathematics,

Nanchang University, Nanchang, Jiangxi 330031, P.R. China

Full list of author information is

available at the end of the article

\begin{abstract}
In this paper, we prove a common fixed point theorem for two pairs of non-self-mappings satisfying the generalized contraction condition of Ćirić type in cone metric spaces. Our result generalizes and extends some recent results related to non-self-mappings in the setting of cone metric spaces.

MSC: $47 \mathrm{H} 10 ; 54 \mathrm{H} 25$
\end{abstract}

Keywords: cone metric spaces; common fixed point; non-self-mappings; contraction condition of Ćirić type

\section{Introduction and preliminaries}

Recently, Huang and Zhang [1] generalized the concept of a metric space, replacing the set of real numbers by ordered Banach space and obtained some fixed point theorems for mappings satisfying different contractive conditions. Subsequently, the study of fixed point theorems in such spaces is followed by some other mathematicians; see [2-24]. The study of fixed point theorems for non-self-mappings in metrically convex metric spaces was initiated by Assad and Kirk [25]. Utilizing the induction method of Assad and Kirk [25], many authors like Assad [26], Ćirić [27-36], Ćirić et al. [37-42], Kumam et al. [43], Hadžić [44], Hadžić and Gajić [45], Imdad and Kumar [46], Rhoades [47, 48] have obtained common fixed point in metrically convex spaces. Recently, Ćirić and Ume [49] defined a wide class of multi-valued non-self-mappings which satisfy a generalized contraction condition and proved a fixed point theorem which generalizes the results of Itoh [50] and Khan [51].

Very recently, Radenović and Rhoades [15] extended the fixed point theorem of Imdad and Kumar [46] for a pair of non-self-mappings to non-normal cone metric spaces. Huang et al. [6] proved a fixed point theorem for four non-self-mappings in cone metric spaces which generalizes the result of Radenović and Rhoades [15]. Janković et al. [9] proved new common fixed point results for a pair of non-self-mappings defined on a closed subset of metrically convex cone metric space which is not necessarily normal by adapting Assad-Kirk's method. Sumitra et al. [20] generalized the fixed point theorems of Ćirić and Ume [49] for a pair of non-self-mappings to non-normal cone metric spaces. In the same 
time, Sumitra et al.'s [20] results extended the results of Radenović and Rhoades [15] and Janković et al. [9]. The aim of this paper is to prove a common fixed point theorem for two pairs of non-self-mappings on cone metric spaces in which the cone need not be normal and the condition is weaker. This result generalizes the result of Sumitra et al. [20] and Huang et al. [6].

Consistent with Huang and Zhang [1], the following definitions and results will be needed in the sequel.

Let $E$ be a real Banach space. A subset $P$ of $E$ is called a cone if and only if:

(a) $P$ is closed, nonempty and $P \neq\{\theta\}$;

(b) $a, b \in R, a, b \geq 0, x, y \in P$ implies $a x+b y \in P$;

(c) $P \cap(-P)=\{\theta\}$.

Given a cone $P \subset E$, we define a partial ordering $\preceq$ with respect to $P$ by $x \preceq y$ if and only if $y-x \in P$. A cone $P$ is called normal if there is a number $K>0$ such that for all $x, y \in E$,

$$
\theta \preceq x \preceq y \text { implies } \quad\|x\| \leq K\|y\| .
$$

The least positive number satisfying the above inequality is called the normal constant of $P$, while $x \ll y$ stands for $y-x \in \operatorname{int} P$ (interior of $P$ ).

Definition 1.1 [1] Let $X$ be a nonempty set. Suppose that the mapping $d: X \times X \rightarrow E$ satisfies:

(d1) $\theta \preceq d(x, y)$ for all $x, y \in X$ and $d(x, y)=\theta$ if and only if $x=y$;

(d2) $d(x, y)=d(y, x)$ for all $x, y \in X$;

(d3) $d(x, y) \preceq d(x, z)+d(z, y)$ for all $x, y, z \in X$.

Then $d$ is called a cone metric on $X$ and $(X, d)$ is called a cone metric space.

The concept of a cone metric space is more general than that of a metric space.

Example 1.1 [1] Let $E=R^{2}, P=\{(x, y) \in E \mid x, y \geq 0\}, X=R$, and $d: X \times X \rightarrow E$ be such that $d(x, y)=(|x-y|, \alpha|x-y|)$, where $\alpha \geq 0$ is a constant. Then $(X, d)$ is a cone metric space.

Definition 1.2 [1] Let $(X, d)$ be a cone metric space. We say that $\left\{x_{n}\right\}$ is:

(e) a Cauchy sequence if for every $c \in E$ with $\theta \ll c$, there is an $N$ such that for all $n, m>N, d\left(x_{n}, x_{m}\right) \ll c ;$

(f) a convergent sequence if for every $c \in E$ with $\theta \ll c$, there is an $N$ such that for all $n>N, d\left(x_{n}, x\right) \ll c$ for some fixed $x \in X$.

A cone metric space $X$ is said to be complete if for every Cauchy sequence in $X$ is convergent in $X$. It is well known that $\left\{x_{n}\right\}$ converges to $x \in X$ if and only if $d\left(x_{n}, x\right) \rightarrow \theta$ as $n \rightarrow \infty$. It is a Cauchy sequence if and only if $d\left(x_{n}, x_{m}\right) \rightarrow \theta(n, m \rightarrow \infty)$.

Remark 1.1 [52] Let $E$ be an ordered Banach (normed) space. Then $c$ is an interior point of $P$, if and only if $[-c, c]$ is a neighborhood of $\theta$.

Corollary 1.1 [16] (1) If $a \preceq b$ and $b \ll c$, then $a \ll c$.

Indeed, $c-a=(c-b)+(b-a) \succeq c-b$ implies $[-(c-a), c-a] \supseteq[-(c-b), c-b]$.

(2) If $a \ll b$ and $b \ll c$, then $a \ll c$.

Indeed, $c-a=(c-b)+(b-a) \succeq c-b$ implies $[-(c-a), c-a] \supseteq[-(c-b), c-b]$.

(3) If $\theta \preceq u \ll c$ for each $c \in \operatorname{int} P$ then $u=\theta$. 
Remark 1.2 $[10,15]$ If $c \in \operatorname{int} P, \theta \preceq a_{n}$, and $a_{n} \rightarrow \theta$, then there exists an $n_{0}$ such that for all $n>n_{0}$ we have $a_{n} \ll c$.

Remark 1.3 [16,17] If $E$ is a real Banach space with cone $P$ and if $a \preceq k a$ where $a \in P$ and $0<k<1$, then $a=\theta$.

Definition 1.3 [2] Let $f$ and $g$ be self-maps on a set $X$ (i.e., $f, g: X \rightarrow X$ ). If $w=f x=g x$ for some $x$ in $X$, then $x$ is called a coincidence point of $f$ and $g$, and $w$ is called a point of coincidence of $f$ and $g$. Self-maps $f$ and $g$ are said to be weakly compatible if they commute at their coincidence point; i.e., if $f x=g x$ for some $x \in X$, then $f g x=g f x$.

\section{Main results}

The following theorem is Sumitra et al.'s [20] generalization of Ćirić and Ume's [49] result in cone metric spaces.

Theorem 2.1 Let $(X, d)$ be a complete cone metric space, $C$ a nonempty closed subset of $X$ such that for each $x \in C$ and $y \notin C$ there exists a point $z \in \partial C$ (the boundary of $C$ ) such that

$$
d(x, z)+d(z, y)=d(x, y)
$$

Suppose that $f, g: C \rightarrow X$ are two non-self-mappings satisfying, for all $x, y \in C$ with $x \neq y$,

$$
d(g x, g y) \preceq \alpha d(f x, f y)+\beta u+\gamma v,
$$

where $u \in\{d(f x, g x), d(f y, g y)\}, v \in\{d(f x, g x)+d(f y, g y), d(f x, g y)+d(f y, g x)\}$, and $\alpha, \beta, \gamma$ are nonnegative real numbers such that

$$
\alpha+2 \beta+3 \gamma+\alpha \gamma<1
$$

Also assume that

(i) $\partial C \subseteq f C, g C \cap C \subseteq f C$,

(ii) $f x \in \partial C$ implies that $g x \in C$,

(iii) $f C$ is closed in $X$.

Then the pair $(f, g)$ has a coincidence point in C. Moreover, if the pair $(f, g)$ is weakly compatible, then $f$ and $g$ have a unique common fixed point in $C$.

Remark 2.1 From the proof of this theorem, it is easy to see that condition (2.2) can be weakened to $\alpha+\beta+2 \gamma<1$.

The purpose of this paper is to extend the above theorem for two pairs of non-selfmappings in cone metric spaces with a weaker condition.

We state and prove our main result as follows.

Theorem 2.2 Let $(X, d)$ be a complete cone metric space, $C$ a nonempty closed subset of $X$ such that for each $x \in C$ and $y \notin C$ there exists a point $z \in \partial C$ such that

$$
d(x, z)+d(z, y)=d(x, y) .
$$


Suppose that $F, G, S, T: C \rightarrow X$ are two pairs ofnon-self-mappings satisfying, for all $x, y \in C$ with $x \neq y$,

$$
d(F x, G y) \preceq \alpha d(T x, S y)+\beta u+\gamma v,
$$

where $u \in\{d(T x, F x), d(S y, G y)\}, v \in\{d(T x, F x)+d(S y, G y), d(T x, G y)+d(S y, F x)\}$, and $\alpha, \beta$, $\gamma$ are nonnegative real numbers such that

$$
\alpha+\beta+2 \gamma<1
$$

\section{Also assume that}

(I) $\partial C \subseteq S C \cap T C, F C \cap C \subseteq S C, G C \cap C \subseteq T C$,

(II) $T x \in \partial C$ implies that $F x \in C, S x \in \partial C$ implies that $G x \in C$,

(III) SC and TC (or FC and GC) are closed in X.

Then

(IV) $(F, T)$ has a point of coincidence,

(V) $(G, S)$ has a point of coincidence.

Moreover, if $(F, T)$ and $(G, S)$ are weakly compatible pairs, then $F, G, S$, and $T$ have a unique common fixed point.

Proof Firstly, we proceed to construct two sequences $\left\{x_{n}\right\}$ and $\left\{y_{n}\right\}$ in the following way.

Let $x \in \partial C$ be arbitrary. Then (due to $\partial C \subseteq T C$ ) there exists a point $x_{0} \in C$ such that $x=T x_{0}$. Since $T x \subseteq \partial C$ implies that $F x \in C$, one concludes that $F x_{0} \in F C \cap C \subseteq S C$. Thus, there exists $x_{1} \in C$ such that $y_{1}=S x_{1}=F x_{0} \in C$. Since $y_{1}=F x_{0}$ there exists a point $y_{2}=G x_{1}$ such that

$$
d\left(y_{1}, y_{2}\right)=d\left(F x_{0}, G x_{1}\right)
$$

Suppose $y_{2} \in C$. Then $y_{2} \in G C \cap C \subseteq T C$, which implies that there exists a point $x_{2} \in C$ such that $y_{2}=T x_{2}$. otherwise, if $y_{2} \notin C$, then there exists a point $p \in \partial C$ such that

$$
d\left(S x_{1}, p\right)+d\left(p, y_{2}\right)=d\left(S x_{1}, y_{2}\right) .
$$

Since $p \in \partial C \subseteq T C$ there exists a point $x_{2} \in C$ with $p=T x_{2}$ so that

$$
d\left(S x_{1}, T x_{2}\right)+d\left(T x_{2}, y_{2}\right)=d\left(S x_{1}, y_{2}\right)
$$

Let $y_{3}=F x_{2}$ be such that $d\left(y_{2}, y_{3}\right)=d\left(G x_{1}, F x_{2}\right)$. Thus, repeating the foregoing arguments, one obtains two sequences $\left\{x_{n}\right\}$ and $\left\{y_{n}\right\}$ such that

(a) $y_{2 n}=G x_{2 n-1}, y_{2 n+1}=F x_{2 n}$,

(b) $y_{2 n} \in C$ implies that $y_{2 n}=T x_{2 n}$ or $y_{2 n} \notin C$ implies that $T x_{2 n} \in \partial C$ and

$$
d\left(S x_{2 n-1}, T x_{2 n}\right)+d\left(T x_{2 n}, y_{2 n}\right)=d\left(S x_{2 n-1}, y_{2 n}\right),
$$

(c) $y_{2 n+1} \in C$ implies that $y_{2 n+1}=S x_{2 n+1}$ or $y_{2 n+1} \notin C$ implies that $S x_{2 n+1} \in \partial C$ and

$$
d\left(T x_{2 n}, S x_{2 n+1}\right)+d\left(S x_{2 n+1}, y_{2 n+1}\right)=d\left(T x_{2 n}, y_{2 n+1}\right) .
$$


We denote

$$
\begin{aligned}
& P_{0}=\left\{T x_{2 i} \in\left\{T x_{2 n}\right\}: T x_{2 i}=y_{2 i}\right\}, \\
& P_{1}=\left\{T x_{2 i} \in\left\{T x_{2 n}\right\}: T x_{2 i} \neq y_{2 i}\right\}, \\
& Q_{0}=\left\{S x_{2 i+1} \in\left\{S x_{2 n+1}\right\}: S x_{2 i+1}=y_{2 i+1}\right\}, \\
& Q_{1}=\left\{S x_{2 i+1} \in\left\{S x_{2 n+1}\right\}: S x_{2 i+1} \neq y_{2 i+1}\right\} .
\end{aligned}
$$

Note that $\left(T x_{2 n}, S x_{2 n+1}\right) \notin P_{1} \times Q_{1}$, as if $T x_{2 n} \in P_{1}$, then $y_{2 n} \neq T x_{2 n}$ and one infers that $T x_{2 n} \in \partial C$, which implies that $y_{2 n+1}=F x_{2 n} \in C$. Hence $y_{2 n+1}=S x_{2 n+1} \in Q_{0}$. Similarly, one can argue that $\left(S x_{2 n-1}, T x_{2 n}\right) \notin Q_{1} \times P_{1}$.

Now, we distinguish the following three cases.

Case 1. If $\left(T x_{2 n}, S x_{2 n+1}\right) \in P_{0} \times Q_{0}$, then from (2.3)

$$
d\left(T x_{2 n}, S x_{2 n+1}\right)=d\left(F x_{2 n}, G x_{2 n-1}\right) \preceq \alpha d\left(T x_{2 n}, S x_{2 n-1}\right)+\beta u_{2 n}+\gamma v_{2 n},
$$

where

$$
\begin{aligned}
u_{2 n} & \in\left\{d\left(T x_{2 n}, F x_{2 n}\right), d\left(S x_{2 n-1}, G x_{2 n-1}\right)\right\}=\left\{d\left(T x_{2 n}, y_{2 n+1}\right), d\left(S x_{2 n-1}, y_{2 n}\right)\right\}, \\
v_{2 n} & \in\left\{d\left(T x_{2 n}, F x_{2 n}\right)+d\left(S x_{2 n-1}, G x_{2 n-1}\right), d\left(T x_{2 n}, G x_{2 n-1}\right)+d\left(S x_{2 n-1}, F x_{2 n}\right)\right\} \\
& =\left\{d\left(T x_{2 n}, y_{2 n+1}\right)+d\left(S x_{2 n-1}, y_{2 n}\right), d\left(S x_{2 n-1}, y_{2 n+1}\right)\right\} .
\end{aligned}
$$

Clearly, there are infinitely many $n$ such that at least one of the following four cases holds:

(1) If $u_{2 n}=d\left(T x_{2 n}, y_{2 n+1}\right)$ and $v_{2 n}=d\left(T x_{2 n}, y_{2 n+1}\right)+d\left(S x_{2 n-1}, y_{2 n}\right)$, then

$$
\begin{aligned}
d\left(T x_{2 n}, S x_{2 n+1}\right) \leq & \alpha d\left(T x_{2 n}, S x_{2 n-1}\right)+\beta d\left(T x_{2 n}, y_{2 n+1}\right) \\
& +\gamma\left(d\left(T x_{2 n}, y_{2 n+1}\right)+d\left(S x_{2 n-1}, y_{2 n}\right)\right) \\
= & \alpha d\left(T x_{2 n}, S x_{2 n-1}\right)+\beta d\left(T x_{2 n}, S x_{2 n+1}\right) \\
& +\gamma d\left(T x_{2 n}, S x_{2 n+1}\right)+\gamma d\left(S x_{2 n-1}, T x_{2 n}\right) .
\end{aligned}
$$

It implies that $d\left(T x_{2 n}, S x_{2 n+1}\right) \preceq \frac{\alpha+\gamma}{1-\beta-\gamma} d\left(S x_{2 n-1}, T x_{2 n}\right)$.

(2) If $u_{2 n}=d\left(T x_{2 n}, y_{2 n+1}\right)$ and $v_{2 n}=d\left(S x_{2 n-1}, y_{2 n+1}\right)$, then

$$
\begin{aligned}
d\left(T x_{2 n}, S x_{2 n+1}\right) \preceq & \alpha d\left(T x_{2 n}, S x_{2 n-1}\right)+\beta d\left(T x_{2 n}, y_{2 n+1}\right)+\gamma d\left(S x_{2 n-1}, y_{2 n+1}\right) \\
\preceq & \alpha d\left(T x_{2 n}, S x_{2 n-1}\right)+\beta d\left(T x_{2 n}, y_{2 n+1}\right) \\
& +\gamma\left(d\left(S x_{2 n-1}, y_{2 n}\right)+d\left(y_{2 n}, y_{2 n+1}\right)\right) \\
= & \alpha d\left(T x_{2 n}, S x_{2 n-1}\right)+\beta d\left(T x_{2 n}, S x_{2 n+1}\right) \\
& +\gamma d\left(S x_{2 n-1}, T x_{2 n}\right)+\gamma d\left(T x_{2 n}, S x_{2 n+1}\right) .
\end{aligned}
$$

It implies that $d\left(T x_{2 n}, S x_{2 n+1}\right) \preceq \frac{\alpha+\gamma}{1-\beta-\gamma} d\left(S x_{2 n-1}, T x_{2 n}\right)$. 
(3) If $u_{2 n}=d\left(S x_{2 n-1}, y_{2 n}\right)$ and $v_{2 n}=d\left(T x_{2 n}, y_{2 n+1}\right)+d\left(S x_{2 n-1}, y_{2 n}\right)$, then

$$
\begin{aligned}
d\left(T x_{2 n}, S x_{2 n+1}\right) \preceq & \alpha d\left(T x_{2 n}, S x_{2 n-1}\right)+\beta d\left(S x_{2 n-1}, y_{2 n}\right) \\
& +\gamma\left(d\left(T x_{2 n}, y_{2 n+1}\right)+d\left(S x_{2 n-1}, y_{2 n}\right)\right) \\
= & \alpha d\left(T x_{2 n}, S x_{2 n-1}\right)+\beta d\left(S x_{2 n-1}, T x_{2 n}\right) \\
& +\gamma d\left(T x_{2 n}, S x_{2 n+1}\right)+\gamma d\left(S x_{2 n-1}, T x_{2 n}\right) .
\end{aligned}
$$

It implies that $d\left(T x_{2 n}, S x_{2 n+1}\right) \preceq \frac{\alpha+\beta+\gamma}{1-\gamma} d\left(S x_{2 n-1}, T x_{2 n}\right)$.

(4) If $u_{2 n}=d\left(S x_{2 n-1}, y_{2 n}\right)$ and $v_{2 n}=d\left(S x_{2 n-1}, y_{2 n+1}\right)$, then

$$
\begin{aligned}
d\left(T x_{2 n}, S x_{2 n+1}\right) \preceq & \alpha d\left(T x_{2 n}, S x_{2 n-1}\right)+\beta d\left(S x_{2 n-1}, y_{2 n}\right)+\gamma d\left(S x_{2 n-1}, y_{2 n+1}\right) \\
\preceq & \alpha d\left(T x_{2 n}, S x_{2 n-1}\right)+\beta d\left(S x_{2 n-1}, y_{2 n}\right) \\
& +\gamma\left(d\left(S x_{2 n-1}, y_{2 n}\right)+d\left(y_{2 n}, y_{2 n+1}\right)\right) \\
= & \alpha d\left(T x_{2 n}, S x_{2 n-1}\right)+\beta d\left(S x_{2 n-1}, T x_{2 n}\right) \\
& +\gamma d\left(S x_{2 n-1}, T x_{2 n}\right)+\gamma d\left(T x_{2 n}, S x_{2 n+1}\right) .
\end{aligned}
$$

It implies that $d\left(T x_{2 n}, S x_{2 n+1}\right) \preceq \frac{\alpha+\beta+\gamma}{1-\gamma} d\left(S x_{2 n-1}, T x_{2 n}\right)$.

From (1), (2), (3), (4) it follows that

$$
d\left(T x_{2 n}, S x_{2 n+1}\right) \preceq \lambda d\left(S x_{2 n-1}, T x_{2 n}\right),
$$

where $\lambda=\max \left\{\frac{\alpha+\gamma}{1-\beta-\gamma}, \frac{\alpha+\beta+\gamma}{1-\gamma}\right\}<1$ by (2.4).

Similarly, if $\left(S x_{2 n+1}, T x_{2 n+2}\right) \in Q_{0} \times P_{0}$, we have

$$
d\left(S x_{2 n+1}, T x_{2 n+2}\right)=d\left(F x_{2 n}, G x_{2 n+1}\right) \preceq \lambda d\left(T x_{2 n}, S x_{2 n+1}\right) .
$$

If $\left(S x_{2 n-1}, T x_{2 n}\right) \in Q_{0} \times P_{0}$, we have

$$
d\left(S x_{2 n-1}, T x_{2 n}\right)=d\left(F x_{2 n-2}, G x_{2 n-1}\right) \preceq \lambda d\left(T x_{2 n-2}, S x_{2 n-1}\right) .
$$

Case 2. If $\left(T x_{2 n}, S x_{2 n+1}\right) \in P_{0} \times Q_{1}$, then $S x_{2 n+1} \in Q_{1}$ and

$$
d\left(T x_{2 n}, S x_{2 n+1}\right)+d\left(S x_{2 n+1}, y_{2 n+1}\right)=d\left(T x_{2 n}, y_{2 n+1}\right),
$$

which in turns yields

$$
d\left(T x_{2 n}, S x_{2 n+1}\right) \preceq d\left(T x_{2 n}, y_{2 n+1}\right)=d\left(y_{2 n}, y_{2 n+1}\right)
$$

and hence

$$
d\left(T x_{2 n}, S x_{2 n+1}\right) \preceq d\left(y_{2 n}, y_{2 n+1}\right)=d\left(F x_{2 n}, G x_{2 n-1}\right)
$$

Now, proceeding as in Case 1, we see that (2.5) holds. 
If $\left(S x_{2 n+1}, T x_{2 n+2}\right) \in Q_{1} \times P_{0}$, then $T x_{2 n} \in P_{0}$. We show that

$$
d\left(S x_{2 n+1}, T x_{2 n+2}\right) \preceq \lambda d\left(T x_{2 n}, S x_{2 n-1}\right) .
$$

Using (2.8), we get

$$
\begin{aligned}
d\left(S x_{2 n+1}, T x_{2 n+2}\right) & \preceq d\left(S x_{2 n+1}, y_{2 n+1}\right)+d\left(y_{2 n+1}, T x_{2 n+2}\right) \\
& =d\left(T x_{2 n}, y_{2 n+1}\right)-d\left(T x_{2 n}, S x_{2 n+1}\right)+d\left(y_{2 n+1}, T x_{2 n+2}\right) .
\end{aligned}
$$

By noting that $T x_{2 n+2}, T x_{2 n} \in P_{0}$, one can conclude that

$$
d\left(y_{2 n+1}, T x_{2 n+2}\right)=d\left(y_{2 n+1}, y_{2 n+2}\right)=d\left(F x_{2 n}, G x_{2 n+1}\right) \preceq \lambda d\left(T x_{2 n}, S x_{2 n+1}\right)
$$

and

$$
d\left(T x_{2 n}, y_{2 n+1}\right)=d\left(y_{2 n}, y_{2 n+1}\right)=d\left(F x_{2 n}, G x_{2 n-1}\right) \preceq \lambda d\left(S x_{2 n-1}, T x_{2 n}\right),
$$

in view of Case 1.

Thus,

$$
d\left(S x_{2 n+1}, T x_{2 n+2}\right) \preceq \lambda d\left(S x_{2 n-1}, T x_{2 n}\right)-(1-\lambda) d\left(T x_{2 n}, S x_{2 n+1}\right) \preceq \lambda d\left(S x_{2 n-1}, T x_{2 n}\right),
$$

and we proved (2.11).

Case 3. If $\left(T x_{2 n}, S x_{2 n+1}\right) \in P_{1} \times Q_{0}$, then $S x_{2 n-1} \in Q_{0}$. We show that

$$
d\left(T x_{2 n}, S x_{2 n+1}\right) \preceq \lambda d\left(S x_{2 n-1}, T x_{2 n-2}\right) .
$$

Since $T x_{2 n} \in P_{1}$, then

$$
d\left(S x_{2 n-1}, T x_{2 n}\right)+d\left(T x_{2 n}, y_{2 n}\right)=d\left(S x_{2 n-1}, y_{2 n}\right)
$$

From this, we get

$$
\begin{aligned}
d\left(T x_{2 n}, S x_{2 n+1}\right) & \preceq d\left(T x_{2 n}, y_{2 n}\right)+d\left(y_{2 n}, S x_{2 n+1}\right) \\
& =d\left(S x_{2 n-1}, y_{2 n}\right)-d\left(S x_{2 n-1}, T x_{2 n}\right)+d\left(y_{2 n}, S x_{2 n+1}\right) .
\end{aligned}
$$

By noting that $S x_{2 n+1}, S x_{2 n-1} \in Q_{0}$, one can conclude that

$$
d\left(y_{2 n}, S x_{2 n+1}\right)=d\left(y_{2 n}, y_{2 n+1}\right)=d\left(F x_{2 n}, G x_{2 n-1}\right) \preceq \lambda d\left(S x_{2 n-1}, T x_{2 n}\right)
$$

and

$$
d\left(S x_{2 n-1}, y_{2 n}\right)=d\left(y_{2 n-1}, y_{2 n}\right)=d\left(F x_{2 n-2}, G x_{2 n-1}\right) \preceq \lambda d\left(S x_{2 n-1}, T x_{2 n-2}\right),
$$

in view of Case 1. 
Thus,

$$
d\left(T x_{2 n}, S x_{2 n+1}\right) \preceq \lambda d\left(S x_{2 n-1}, T x_{2 n-2}\right)-(1-\lambda) d\left(S x_{2 n-1}, T x_{2 n}\right) \preceq \lambda d\left(S x_{2 n-1}, T x_{2 n-2}\right),
$$

and we proved (2.15).

Similarly, if $\left(S x_{2 n+1}, T x_{2 n+2}\right) \in Q_{0} \times P_{1}$, then $T x_{2 n+2} \in P_{1}$, and

$$
d\left(S x_{2 n+1}, T x_{2 n+2}\right)+d\left(T x_{2 n+2}, y_{2 n+2}\right)=d\left(S x_{2 n+1}, y_{2 n+2}\right) .
$$

From this, we have

$$
\begin{aligned}
d\left(S x_{2 n+1}, T x_{2 n+2}\right) & \preceq d\left(S x_{2 n+1}, y_{2 n+2}\right)+d\left(y_{2 n+2}, T x_{2 n+2}\right) \\
& \preceq d\left(S x_{2 n+1}, y_{2 n+2}\right)+d\left(S x_{2 n+1}, y_{2 n+2}\right)-d\left(S x_{2 n+1}, T x_{2 n+2}\right) \\
& =2 d\left(S x_{2 n+1}, y_{2 n+2}\right)-d\left(S x_{2 n+1}, T x_{2 n+2}\right) \\
\Rightarrow \quad d\left(S x_{2 n+1},\right. & \left.T x_{2 n+2}\right) \preceq d\left(S x_{2 n+1}, y_{2 n+2}\right) .
\end{aligned}
$$

By noting that $S x_{2 n+1} \in Q_{0}$, one can conclude that

$$
d\left(S x_{2 n+1}, T x_{2 n+2}\right) \preceq d\left(S x_{2 n+1}, y_{2 n+2}\right)=d\left(F x_{2 n}, G x_{2 n+1}\right) \preceq \lambda d\left(T x_{2 n}, S x_{2 n+1}\right),
$$

in view of Case 1.

Thus, in all cases (1)-(3), there exists $w_{2 n} \in\left\{d\left(S x_{2 n-1}, T x_{2 n}\right), d\left(T x_{2 n-2}, S x_{2 n-1}\right)\right\}$ such that

$$
d\left(T x_{2 n}, S x_{2 n+1}\right) \preceq \lambda w_{2 n}
$$

and there exists $w_{2 n+1} \in\left\{d\left(S x_{2 n-1}, T x_{2 n}\right), d\left(T x_{2 n}, S x_{2 n+1}\right)\right\}$ such that

$$
d\left(S x_{2 n+1}, T x_{2 n+2}\right) \preceq \lambda w_{2 n+1} .
$$

Following the procedure of Assad and Kirk [25], it can easily be shown by induction that, for $n \geq 1$, there exists $w_{2} \in\left\{d\left(T x_{0}, S x_{1}\right), d\left(S x_{1}, T x_{2}\right)\right\}$ such that

$$
d\left(T x_{2 n}, S x_{2 n+1}\right) \preceq \lambda^{n-\frac{1}{2}} w_{2} \text { and } d\left(S x_{2 n+1}, T x_{2 n+2}\right) \preceq \lambda^{n} w_{2}
$$

From (2.21) and by the triangle inequality, for $n>m$ we have

$$
\begin{aligned}
d\left(T x_{2 n}, S x_{2 m+1}\right) & \leq d\left(T x_{2 n}, S x_{2 n-1}\right)+d\left(S x_{2 n-1}, T x_{2 n-2}\right)+\cdots+d\left(T x_{2 m+2}, S x_{2 m+1}\right) \\
& \preceq\left(\lambda^{m}+\lambda^{m+\frac{1}{2}}+\cdots+\lambda^{n-1}\right) w_{2} \preceq \frac{\lambda^{m}}{1-\sqrt{\lambda}} w_{2} \rightarrow \theta, \quad \text { as } m \rightarrow \infty .
\end{aligned}
$$

From Remark 1.2 and Corollary 1.1(1), it follows that $d\left(T x_{2 n}, S x_{2 m+1}\right) \ll c$.

Thus, the sequence $\left\{T x_{0}, S x_{1}, T x_{2}, S x_{3}, \ldots, S x_{2 n-1}, T x_{2 n}, S x_{2 n-1}, \ldots\right\}$ is a Cauchy sequence. Then, as noted in [45], there exists at least one subsequence, $\left\{T x_{2 n_{k}}\right\}$ or $\left\{S x_{2 n_{k}+1}\right\}$, which is contained in $P_{0}$ or $Q_{0}$, respectively, and one finds its limit $z \in C$. Furthermore, subsequences $\left\{T x_{2 n_{k}}\right\}$ and $\left\{S x_{2 n_{k}+1}\right\}$ both converge to $z \in C$ as $C$ is a closed subset of complete cone metric space $(X, d)$. We assume that there exists a subsequence $\left\{T x_{2 n_{k}}\right\} \subseteq P_{0}$ 
for each $k \in N$, then $T x_{2 n_{k}}=y_{2 n_{k}}=G x_{2 n_{k}-1} \in C \cap G C \subseteq T C$ Since $T C$ as well as $S C$ are closed in $X$ and $\left\{T x_{2 n_{k}}\right\}$ is Cauchy in $T C$, it converges to a point $z \in T C$. Let $w \in T^{-1} z$, then $T w=z$. Similarly, $\left\{T x_{2 n_{k}}\right\}$ and $\left\{S x_{2 n_{k}+1}\right\}$ being a subsequence of a Cauchy sequence, $\left\{T x_{0}, S x_{1}, T x_{2}, S x_{3}, \ldots, S x_{2 n-1}, T x_{2 n}, S x_{2 n-1}, \ldots\right\}$ also converges to $z$ as $S C$ is closed. Let $\theta \ll c$, then $d\left(z, S x_{2 n_{k}-1}\right) \ll \frac{c}{2 \frac{\alpha+\gamma}{1-\beta-\gamma}}$, where $\alpha, \beta, \gamma$ are nonnegative real numbers with $\alpha+\beta+2 \gamma<1$.

Using (2.3), one can write

$$
\begin{aligned}
d(F w, z) & \leq d\left(F w, G x_{2 n_{k}-1}\right)+d\left(G x_{2 n_{k}-1}, z\right) \\
& \preceq \alpha d\left(T w, S x_{2 n_{k}-1}\right)+\beta u_{w}+\gamma v_{w}+d\left(G x_{2 n_{k}-1}, z\right) \\
& =\alpha d\left(z, S x_{2 n_{k}-1}\right)+\beta u_{w}+\gamma v_{w}+d\left(G x_{2 n_{k}-1}, z\right),
\end{aligned}
$$

where

$$
\begin{aligned}
u_{w} & \in\left\{d(T w, F w), d\left(S x_{2 n_{k}-1}, G x_{2 n_{k}-1}\right)\right\}=\left\{d(z, F w), d\left(S x_{2 n_{k}-1}, G x_{2 n_{k}-1}\right)\right\}, \\
v_{w} & \in\left\{d(T w, F w)+d\left(S x_{2 n_{k}-1}, G x_{2 n_{k}-1}\right), d\left(T w, G x_{2 n_{k}-1}\right)+d\left(F w, S x_{2 n_{k}-1}\right)\right\} \\
& =\left\{d(z, F w)+d\left(S x_{2 n_{k}-1}, G x_{2 n_{k}-1}\right), d\left(z, G x_{2 n_{k}-1}\right)+d\left(F w, S x_{2 n_{k}-1}\right)\right\} .
\end{aligned}
$$

Let $\theta \ll c$. Clearly at least one of the following four cases holds for infinitely many $n$ :

(1) If $u_{w}=d(z, F w)$ and $v_{w}=d(z, F w)+d\left(S x_{2 n_{k}-1}, G x_{2 n_{k}-1}\right)$, then

$$
\begin{aligned}
d(F w, z) \subseteq & \alpha d\left(z, S x_{2 n_{k}-1}\right)+\beta d(z, F w) \\
& +\gamma\left(d(z, F w)+d\left(S x_{2 n_{k}-1}, G x_{2 n_{k}-1}\right)\right)+d\left(G x_{2 n_{k}-1}, z\right) \\
\preceq & \alpha d\left(z, S x_{2 n_{k}-1}\right)+\beta d(z, F w)+\gamma d(z, F w) \\
& +\gamma\left(d\left(S x_{2 n_{k}-1}, z\right)+d\left(z, G x_{2 n_{k}-1}\right)\right)+d\left(G x_{2 n_{k}-1}, z\right) \\
= & (\alpha+\gamma) d\left(z, S x_{2 n_{k}-1}\right)+(\beta+\gamma) d(z, F w)+(\gamma+1) d\left(G x_{2 n_{k}-1}, z\right) \\
\Rightarrow \quad d(F w, z) \preceq & \frac{\alpha+\gamma}{1-\beta-\gamma} d\left(z, S x_{2 n_{k}-1}\right)+\frac{\gamma+1}{1-\beta-\gamma} d\left(G x_{2 n_{k}-1}, z\right) \\
\ll & \frac{\alpha+\gamma}{1-\beta-\gamma} \frac{c}{2 \frac{\alpha+\gamma}{1-\beta-\gamma}}+\frac{\gamma+1}{1-\beta-\gamma} \frac{c}{2 \frac{\gamma+1}{1-\beta-\gamma}}=c .
\end{aligned}
$$

(2) If $u_{w}=d(z, F w)$ and $v_{w}=d\left(z, G x_{2 n_{k}-1}\right)+d\left(F w, S x_{2 n_{k}-1}\right)$, then

$$
\begin{aligned}
& d(F w, z) \preceq \alpha d\left(z, S x_{2 n_{k}-1}\right)+\beta d(z, F w) \\
&+\gamma\left(d\left(z, G x_{2 n_{k}-1}\right)+d\left(F w, S x_{2 n_{k}-1}\right)\right)+d\left(G x_{2 n_{k}-1}, z\right) \\
& \preceq \alpha d\left(z, S x_{2 n_{k}-1}\right)+\beta d(z, F w)+\gamma d\left(z, G x_{2 n_{k}-1}\right) \\
&+\gamma\left(d(F w, z)+d\left(z, S x_{2 n_{k}-1}\right)\right)+d\left(G x_{2 n_{k}-1}, z\right) \\
&=(\alpha+\gamma) d\left(z, S x_{2 n_{k}-1}\right)+(\beta+\gamma) d(z, F w)+(\gamma+1) d\left(G x_{2 n_{k}-1}, z\right) \\
& \Rightarrow \quad d(F w, z) \preceq \frac{\alpha+\gamma}{1-\beta-\gamma} d\left(z, S x_{2 n_{k}-1}\right)+\frac{\gamma+1}{1-\beta-\gamma} d\left(G x_{2 n_{k}-1}, z\right) \\
& \ll \frac{\alpha+\gamma}{1-\beta-\gamma} \frac{c}{2 \frac{\alpha+\gamma}{1-\beta-\gamma}}+\frac{\gamma+1}{1-\beta-\gamma} \frac{c}{2 \frac{\gamma+1}{1-\beta-\gamma}}=c .
\end{aligned}
$$


(3) If $u_{w}=d\left(S x_{2 n_{k}-1}, G x_{2 n_{k}-1}\right)$ and $v_{w}=d(z, F w)+d\left(S x_{2 n_{k}-1}, G x_{2 n_{k}-1}\right)$, then

$$
\begin{aligned}
d(F w, z) \preceq & \alpha d\left(z, S x_{2 n_{k}-1}\right)+\beta d\left(S x_{2 n_{k}-1}, G x_{2 n_{k}-1}\right) \\
& +\gamma\left(d(z, F w)+d\left(S x_{2 n_{k}-1}, G x_{2 n_{k}-1}\right)\right)+d\left(G x_{2 n_{k}-1}, z\right) \\
\preceq & \alpha d\left(z, S x_{2 n_{k}-1}\right)+\beta\left(d\left(S x_{2 n_{k}-1}, z\right)+d\left(z, G x_{2 n_{k}-1}\right)\right)+\gamma d(z, F w) \\
& +\gamma\left(d\left(S x_{2 n_{k}-1}, z\right)+d\left(z, G x_{2 n_{k}-1}\right)\right)+d\left(G x_{2 n_{k}-1}, z\right) \\
= & (\alpha+\beta+\gamma) d\left(z, S x_{2 n_{k}-1}\right)+\gamma d(z, F w)+(\beta+\gamma+1) d\left(G x_{2 n_{k}-1}, z\right) \\
\Rightarrow \quad d(F w, z) \preceq & \frac{\alpha+\beta+\gamma}{1-\gamma} d\left(z, S x_{2 n_{k}-1}\right)+\frac{\beta+\gamma+1}{1-\gamma} d\left(G x_{2 n_{k}-1}, z\right) \\
\ll & \frac{\alpha+\beta+\gamma}{1-\gamma} \frac{c}{2 \frac{\alpha+\beta+\gamma}{1-\gamma}}+\frac{\beta+\gamma+1}{1-\gamma} \frac{c}{2 \frac{\beta+\gamma+1}{1-\gamma}}=c .
\end{aligned}
$$

(4) If $u_{w}=d\left(S x_{2 n_{k}-1}, G x_{2 n_{k}-1}\right)$ and $v_{w}=d\left(z, G x_{2 n_{k}-1}\right)+d\left(F w, S x_{2 n_{k}-1}\right)$, then

$$
\begin{aligned}
d(F w, z) \preceq & \alpha d\left(z, S x_{2 n_{k}-1}\right)+\beta d\left(S x_{2 n_{k}-1}, G x_{2 n_{k}-1}\right) \\
& +\gamma\left(d\left(z, G x_{2 n_{k}-1}\right)+d\left(F w, S x_{2 n_{k}-1}\right)\right)+d\left(G x_{2 n_{k}-1}, z\right) \\
\preceq & \alpha d\left(z, S x_{2 n_{k}-1}\right)+\beta\left(d\left(S x_{2 n_{k}-1}, z\right)+d\left(z, G x_{2 n_{k}-1}\right)\right)+\gamma d\left(z, G x_{2 n_{k}-1}\right) \\
& +\gamma\left(d(F w, z)+d\left(z, S x_{2 n_{k}-1}\right)\right)+d\left(G x_{2 n_{k}-1}, z\right) \\
= & (\alpha+\beta+\gamma) d\left(z, S x_{2 n_{k}-1}\right)+\gamma d(z, F w)+(\beta+\gamma+1) d\left(G x_{2 n_{k}-1}, z\right) \\
\Rightarrow \quad d(F w, z) \preceq & \frac{\alpha+\beta+\gamma}{1-\gamma} d\left(z, S x_{2 n_{k}-1}\right)+\frac{\beta+\gamma+1}{1-\gamma} d\left(G x_{2 n_{k}-1}, z\right) \\
\ll & \frac{\alpha+\beta+\gamma}{1-\gamma} \frac{c}{2 \frac{\alpha+\beta+\gamma}{1-\gamma}}+\frac{\beta+\gamma+1}{1-\gamma} \frac{c}{2 \frac{\beta+\gamma+1}{1-\gamma}}=c .
\end{aligned}
$$

In all cases we obtain $d(F w, z) \ll c$ for each $c \in$ int $P$. Using Corollary 1.1(3) it follows that $d(F w, z)=\theta$ or $F w=z$. Thus, $F w=z=T w$, that is, $z$ is a coincidence point of $F, T$.

Further, since Cauchy sequence $\left\{T x_{0}, S x_{1}, T x_{2}, S x_{3}, \ldots, S x_{2 n-1}, T x_{2 n}, S x_{2 n-1}, \ldots\right\}$ converges to $z \in C$ and $z=F w, z \in F C \cap C \subseteq S C$, there exists $b \in C$ such that $S b=z$. Again using (2.3), we get

$$
d(S b, G b)=d(z, G b)=d(F w, G b) \preceq \alpha d(T w, S b)+\beta u_{w}+\gamma v_{w}=\beta u_{w}+\gamma v_{w},
$$

where

$$
\begin{aligned}
u_{w} & \in\{d(T w, F w), d(S b, G b)\}=\{\theta, d(S b, G b)\}, \\
v_{w} & \in\{d(T w, F w)+d(S b, G b), d(T w, G b)+d(S b, F w)\} \\
& =\{d(S b, G b), d(z, G b)\}=\{d(S b, G b)\} .
\end{aligned}
$$

Hence, we get the following cases:

$$
d(S b, G b) \preceq \beta \theta+\gamma d(S b, G b)=\gamma d(S b, G b) \quad \text { and } \quad d(S b, G b) \preceq(\beta+\gamma) d(S b, G b) .
$$


Since $0 \leq \gamma \leq \beta+\gamma<1-\alpha-\gamma \leq 1$, using Remark 1.3 and Corollary 1.1(3), it follows that $S b=G b$, therefore, $S b=z=G b$, that is, $z$ is a coincidence point of (G, $S$ ).

In case $F C$ and $G C$ are closed in $X$, then $z \in F C \cap C \subseteq S C$ or $z \in G C \cap C \subseteq T C$. The analogous arguments establish (IV) and (V). If we assume that there exists a subsequence $\left\{S x_{2 n_{k}+1}\right\} \subseteq Q_{0}$ with $T C$ as well $S C$ are closed in $X$, then noting that $\left\{S x_{2 n_{k}+1}\right\}$ is a Cauchy sequence in $S C$, the foregoing arguments establish (IV) and (V).

Suppose now that $(F, T)$ and $(G, S)$ are weakly compatible pairs, then

$$
\begin{array}{lll}
z=F w=T w \quad & \Rightarrow & F z=F T w=T F w=T z \quad \text { and } \\
z=G b=S b \quad \Rightarrow \quad G z=G S b=S G b=S z .
\end{array}
$$

Then, from (2.3),

$$
d(F z, z)=d(F z, G b) \preceq \alpha d(T z, S b)+\beta u+\gamma v=\alpha d(F z, z)+\beta u+\gamma v,
$$

where

$$
\begin{aligned}
u & \in\{d(T z, F z), d(S b, G b)\}=\{d(F z, F z), d(z, z)\}=\{\theta\}, \\
v & \in\{d(T z, F z)+d(S b, G b), d(T z, G b)+d(S b, F z)\} \\
& =\{\theta, d(F z, z)+d(z, F z)\}=\{\theta, 2 d(F z, z)\} .
\end{aligned}
$$

Hence, we get the following cases:

$$
d(F z, z) \preceq \alpha d(F z, z) \quad \text { and } \quad d(F z, z) \preceq \alpha d(F z, z)+2 \gamma d(F z, z)=(\alpha+2 \gamma) d(z, F z) .
$$

Since $0 \leq \alpha \leq \alpha+2 \gamma<1-\beta \leq 1$, using Remark 1.3 and Corollary 1.1(3), it follows that $F z=z$. Thus, $F z=z=T z$.

Similarly, we can prove $G z=z=S z$. Therefore $z=F z=G z=S z=T z$, that is, $z$ is a common fixed point of $F, G, S$, and $T$.

Uniqueness of the common fixed point follows easily from (2.3).

The following example shows that in general $F, G, S$, and $T$ satisfying the hypotheses of Theorem 2.2 need not have a common coincidence justifying the two separate conclusions (IV) and (V).

Example 2.1 Let $E=C^{1}([0,1], R), P=\{\varphi \in E: \varphi(t) \geq 0, t \in[0,1]\}, X=[0,+\infty), C=[0,2]$, and $d: X \times X \rightarrow E$ defined by $d(x, y)=|x-y| \varphi$, where $\varphi \in P$ is a fixed function, e.g., $\varphi(t)=e^{t}$. Then $(X, d)$ is a complete cone metric space with a non-normal cone having a nonempty interior. Define $F, G, S$, and $T: C \rightarrow X$ as

$$
F x=x+\frac{4}{5}, \quad G x=x^{2}+\frac{4}{5}, \quad T x=5 x \quad \text { and } \quad S x=5 x^{2}, \quad x \in C .
$$

Since $\partial C=\{0,2\}$. Clearly, for each $x \in C$ and $y \notin C$ there exists a point $z=2 \in \partial C$ such that $d(x, z)+d(z, y)=d(x, y)$. Further, $S C \cap T C=[0,20] \cap[0,10]=[0,10] \supset\{0,2\}=\partial C$, 
$F C \cap C=\left[\frac{4}{5}, \frac{14}{5}\right] \cap[0,2]=\left[\frac{4}{5}, 2\right] \subset S C, G C \cap C=\left[\frac{4}{5}, \frac{24}{5}\right] \cap[0,2]=\left[\frac{4}{5}, 2\right] \subset T C$, and $, S C, T C$, $F C$, and $G C$ are closed in $X$.

Also,

$$
\begin{aligned}
& T 0=0 \in \partial C \quad \Rightarrow \quad F 0=\frac{4}{5} \in C, \\
& S 0=0 \in \partial C \quad \Rightarrow \quad G 0=\frac{4}{5} \in C, \\
& T\left(\frac{2}{5}\right)=2 \in \partial C \quad \Rightarrow \quad F\left(\frac{2}{5}\right)=\frac{6}{5} \in C, \\
& S\left(\sqrt{\frac{2}{5}}\right)=2 \in \partial C \quad \Rightarrow \quad G\left(\sqrt{\frac{2}{5}}\right)=\frac{6}{5} \in C .
\end{aligned}
$$

Moreover, for each $x, y \in C$,

$$
d(F x, G y)=\left|x-y^{2}\right| \varphi=\frac{1}{5} d(T x, S y),
$$

that is, (2.3) is satisfied with $\alpha=\frac{1}{5}, \beta=\gamma=0$.

Obviously, $1=T\left(\frac{1}{5}\right)=F\left(\frac{1}{5}\right) \neq \frac{1}{5}$ and $1=S\left(\frac{1}{\sqrt{5}}\right)=G\left(\frac{1}{\sqrt{5}}\right) \neq \frac{1}{\sqrt{5}}$. Notice that two separate coincidence points are not common fixed points as $F T\left(\frac{1}{5}\right) \neq T F\left(\frac{1}{5}\right)$ and $S G\left(\frac{1}{\sqrt{5}}\right) \neq G S\left(\frac{1}{\sqrt{5}}\right)$, which shows the necessity of the weakly compatibility property in Theorem 2.2 .

Next, we furnish an illustrate example in support of our result. In doing so, we are essentially inspired by Imdad and Kumar [46].

Example 2.2 Let $E=C^{1}([0,1], R), P=\{\varphi \in E: \varphi(t) \geq 0, t \in[0,1]\}, X=[1,+\infty), C=[1,3]$, and $d: X \times X \rightarrow E$ defined by $d(x, y)=|x-y| \varphi$, where $\varphi \in P$ is a fixed function, e.g., $\varphi(t)=e^{t}$. Then $(X, d)$ is a complete cone metric space with a non-normal cone having the nonempty interior. Define $F, G, S$, and $T: C \rightarrow X$ as

$$
\begin{aligned}
& F x=\left\{\begin{array}{ll}
x^{2} & \text { if } 1 \leq x \leq 2, \\
2 & \text { if } 2<x \leq 3,
\end{array} \quad T x= \begin{cases}4 x^{4}-3 & \text { if } 1 \leq x \leq 2, \\
13 & \text { if } 2<x \leq 3,\end{cases} \right. \\
& G x=\left\{\begin{array}{ll}
x^{3} & \text { if } 1 \leq x \leq 2, \\
2 & \text { if } 2<x \leq 3
\end{array} \text { and } \quad S x= \begin{cases}4 x^{6}-3 & \text { if } 1 \leq x \leq 2, \\
13 & \text { if } 2<x \leq 3 .\end{cases} \right.
\end{aligned}
$$

Since $\partial C=\{1,3\}$. Clearly, for each $x \in C$ and $y \notin C$ there exists a point $z=3 \in \partial C$ such that $d(x, z)+d(z, y)=d(x, y)$. Further, $S C \cap T C=[1,253] \cap[1,61]=[1,61] \supset\{1,3\}=\partial C$, $F C \cap C=[1,4] \cap[1,3]=[1,3] \subset S C$, and $G C \cap C=[1,8] \cap[1,3]=[1,3] \subset T C$.

Also,

$$
\begin{aligned}
& T 1=1 \in \partial C \quad \Rightarrow \quad F 1=1 \in C, \\
& S 1=1 \in \partial C \quad \Rightarrow \quad G 1=1 \in C, \\
& T\left(\sqrt[4]{\frac{3}{2}}\right)=3 \in \partial C \quad \Rightarrow \quad F\left(\sqrt[4]{\frac{3}{2}}\right)=\sqrt{\frac{3}{2}} \in C, \\
& S\left(\sqrt[6]{\frac{3}{2}}\right)=3 \in \partial C \quad \Rightarrow \quad G\left(\sqrt[6]{\frac{3}{2}}\right)=\sqrt{\frac{3}{2}} \in C .
\end{aligned}
$$


Moreover, if $x \in[1,2]$ and $y \in[2,3]$, then

$$
d(F x, G y)=\left|x^{2}-2\right| \varphi=\frac{\left|x^{4}-4\right|}{\left|x^{2}+2\right|} \varphi=\frac{4\left|x^{4}-4\right|}{4\left|x^{2}+2\right|} \varphi=\frac{1}{4\left(x^{2}+2\right)} d(T x, S y) .
$$

Next, if $x, y \in(2,3]$, then

$$
d(F x, G y)=0=\alpha \cdot d(T x, S y) .
$$

Finally, if $x, y \in[1,2]$, then

$$
d(F x, G y)=\left|x^{2}-y^{3}\right| \varphi=\frac{\left|x^{4}-y^{6}\right|}{\left|x^{2}+y^{3}\right|} \varphi=\frac{4\left|x^{4}-y^{6}\right|}{4\left|x^{2}+y^{3}\right|} \varphi=\frac{1}{4\left(x^{2}+y^{3}\right)} d(T x, S y) .
$$

Therefore, condition (2.3) is satisfied if we choose $\alpha=\max \left\{\frac{1}{4\left(x^{2}+2\right)}, \frac{1}{4\left(x^{2}+y^{3}\right)}\right\} \in\left(0, \frac{1}{4}\right), \beta=$ $\gamma=0$. Moreover, 1 is a point of coincidence as $T 1=F 1$ as well as $S 1=G 1$, whereas both pairs $(F, T)$ and $(G, S)$ are weakly compatible as $T F 1=1=F T 1$ and $S G 1=1=G S 1$. Also, $S C, T C, F C$, and $G C$ are closed in $X$. Thus, all the conditions of Theorem 2.2 are satisfied and 1 is the unique common fixed point of $F, G, S$, and $T$. One may note that 1 is also a point of coincidence for both pairs $(F, T)$ and $(G, S)$.

Remark 2.2 1. Setting $G=F=g$ and $T=S=f$ in Theorem 2.2, one deduces Theorem 2.1 due to Sumitra et al. [20] with weaker condition.

2. Setting $G=F=g$ and $T=S=I_{X}$ in Theorem 2.2, we obtain the following result.

Corollary 2.1 Let $(X, d)$ be a complete cone metric space, and $C$ a nonempty closed subset of $X$ such that for each $x \in C$ and $y \notin C$ there exists a point $z \in \partial C$ such that

$$
d(x, z)+d(z, y)=d(x, y)
$$

Suppose that $g: C \rightarrow X$ satisfies, for all $x, y \in C$ with $x \neq y$,

$$
d(g x, g y) \preceq \alpha d(x, y)+\beta u+\gamma
$$

where

$$
u \in\{d(x, g x), d(y, g y)\}, \quad v \in\{d(x, g x)+d(y, g y), d(x, g y)+d(y, g x)\},
$$

and $\alpha, \beta, \gamma$ are nonnegative real numbers such that $\alpha+\beta+2 \gamma<1$ and $g$ has the additional property that if, for each $x \in \partial C, g x \in C$, then $g$ has a unique fixed point in $C$.

Corollary 2.2 Let $(X, d)$ be a complete cone metric space, $C$ a nonempty closed subset of $X$ such that for each $x \in C$ and $y \notin C$ there exists a point $z \in \partial C$ such that

$$
d(x, z)+d(z, y)=d(x, y) .
$$

Let $F, G, S, T: C \rightarrow X$ be such that

$$
d(F x, G y) \preceq \alpha d(T x, S y)
$$

for some $\alpha \in(0,1)$ and for all $x, y \in C$ with $x \neq y$. 
Suppose, further, that $F, G, S, T$, and $C$ satisfy the following conditions:

(I) $\partial C \subseteq S C \cap T C, F C \cap C \subseteq S C, G C \cap C \subseteq T C$,

(II) $T x \subseteq \partial C \Rightarrow F x \in C, S x \subseteq \partial C \Rightarrow G x \in C$,

(III) SC and TC (or FC and GC) are closed in $X$.

Then

(IV) $(F, T)$ has a point of coincidence,

(V) $(G, S)$ has a point of coincidence.

Moreover, if $(F, T)$ and $(G, S)$ are weakly compatible pairs, then $F, G, S$, and $T$ have a unique common fixed point.

Corollary 2.3 Let $(X, d)$ be a complete cone metric space, $C$ a nonempty closed subset of $X$ such that for each $x \in C$ and $y \notin C$ there exists a point $z \in \partial C$ such that

$$
d(x, z)+d(z, y)=d(x, y) .
$$

Let $F, G, S, T: C \rightarrow X$ be such that

$$
d(F x, G y) \preceq \gamma(d(T x, F x)+d(S y, G y))
$$

for some $\gamma \in(0,1 / 2)$ and for all $x, y \in C$ with $x \neq y$.

Suppose, further, that $F, G, S, T$, and $C$ satisfy the following conditions:

(I) $\partial C \subseteq S C \cap T C, F C \cap C \subseteq S C, G C \cap C \subseteq T C$,

(II) $T x \subseteq \partial C \Rightarrow F x \in C, S x \subseteq \partial C \Rightarrow G x \in C$,

(III) $S C$ and TC (or FC and GC) are closed in $X$.

Then

(IV) $(F, T)$ has a point of coincidence,

(V) $(G, S)$ has a point of coincidence.

Moreover, if $(F, T)$ and $(G, S)$ are weakly compatible pairs, then $F, G, S$, and $T$ have a unique common fixed point.

Corollary 2.4 Let $(X, d)$ be a complete cone metric space, $C$ a nonempty closed subset of $X$ such that for each $x \in C$ and $y \notin C$ there exists a point $z \in \partial C$ such that

$$
d(x, z)+d(z, y)=d(x, y) .
$$

Let $F, G, S, T: C \rightarrow X$ be such that

$$
d(F x, G y) \preceq \gamma(d(T x, F y)+d(F x, S y))
$$

for some $\gamma \in(0,1 / 2)$ and for all $x, y \in C$ with $x \neq y$.

Suppose, further, that $F, G, S, T$, and $C$ satisfy the following conditions:

(I) $\partial C \subseteq S C \cap T C, F C \cap C \subseteq S C, G C \cap C \subseteq T C$,

(II) $T x \subseteq \partial C \Rightarrow F x \in C, S x \subseteq \partial C \Rightarrow G x \in C$,

(III) $S C$ and $T C$ (or FC and GC) are closed in $X$.

Then

(IV) $(F, T)$ has a point of coincidence,

(V) $(G, S)$ has a point of coincidence. 
Moreover, if $(F, T)$ and $(G, S)$ are weakly compatible pairs, then $F, G, S$, and $T$ have a unique common fixed point.

Remark 2.3 Setting $G=F=f$ and $T=S=g$ in Corollaries 2.2-2.4, we obtain the following results.

Corollary 2.5 Let $(X, d)$ be a complete cone metric space, $C$ a nonempty closed subset of $X$ such that for each $x \in C$ and $y \notin C$ there exists a point $z \in \partial C$ such that

$$
d(x, z)+d(z, y)=d(x, y)
$$

Letf $, g: C \rightarrow X$ be such that

$$
d(f x, f y) \preceq \alpha d(g x, g y)
$$

for some $\alpha \in(0,1)$ and for all $x, y \in C$. Suppose, further, that $f, g$, and $C$ satisfy the following conditions:

(I) $\partial C \subseteq g C, f C \cap C \subseteq g C$,

(II) $g x \subseteq \partial C \Rightarrow f x \in C$,

(III) $g C$ is closed in $X$.

Then the pair $(f, g)$ has a coincidence point in C. Moreover, if the pair $(f, g)$ is weakly compatible, then $f$ and $g$ have a unique common fixed point in $C$.

Corollary 2.6 Let $(X, d)$ be a complete cone metric space, $C$ a nonempty closed subset of $X$ such that for each $x \in C$ and $y \notin C$ there exists a point $z \in \partial C$ (the boundary of $C$ ) such that

$$
d(x, z)+d(z, y)=d(x, y) .
$$

Let $f, g: C \rightarrow X$ be such that

$$
d(f x, f y) \preceq \gamma(d(f x, g x)+d(f y, g y))
$$

for some $\gamma \in(0,1 / 2)$ and for all $x, y \in C$. Suppose, further, that $f, g$, and $C$ satisfy the following conditions:

(I) $\partial C \subseteq g C, f C \cap C \subseteq g C$,

(II) $g x \subseteq \partial C \Rightarrow f x \in C$,

(III) $g C$ is closed in $X$.

Then the pair $(f, g)$ has a coincidence point in C. Moreover, if the pair $(f, g)$ is weakly compatible, then $f$ and $g$ have a unique common fixed point in $C$.

Corollary 2.7 Let $(X, d)$ be a complete cone metric space, $C$ a nonempty closed subset of $X$ such that for each $x \in C$ and $y \notin C$ there exists a point $z \in \partial C$ (the boundary of $C$ ) such that

$$
d(x, z)+d(z, y)=d(x, y) .
$$


Let $f, g: C \rightarrow X$ be such that

$$
d(f x, f y) \preceq \gamma(d(f x, g y)+d(f y, g x))
$$

for some $\gamma \in(0,1 / 2)$ and for all $x, y \in C$. Suppose, further, that $f, g$, and $C$ satisfy the following conditions:

(I) $\partial C \subseteq g C, f C \cap C \subseteq g C$,

(II) $g x \subseteq \partial C \Rightarrow f x \in C$,

(III) $g C$ is closed in $X$.

Then the pair $(f, g)$ has a coincidence point in C. Moreover, if the pair $(f, g)$ is weakly compatible, then $f$ and $g$ have a unique common fixed point in $C$.

Remark 2.4 Corollaries 2.5-2.7 are the corresponding theorems of Abbas and Jungck from [2] in the case that $f, g$ are non-self-mappings.

There have been a number of papers written about fixed points for non-self-maps. One of the most general papers involving two maps is that in [30]. For cone metric spaces, the four-function analog of this result would have the contractive condition.

Suppose that $F, G, S, T: C \rightarrow X$ are two pairs of non-self-mappings satisfying, for all $x, y \in C$ with $x \neq y$,

$$
d(F x, G y) \preceq h w,
$$

where $w \in\left\{\frac{d(T x, S y)}{a}, d(T x, F x), d(S y, G y), \frac{d(T x, G y)+d(S y, F x)}{a+h}\right\}, a$ is a positive real number satisfying $a \geq 1+\frac{2 h^{2}}{1+h}$ and $0<h<1$.

Note that if $F, G, S$, and $T$ satisfy condition (2.28), then $F, G, S$, and $T$ satisfy condition (2.3), but the implication is not reversible.

Indeed, there are four cases to consider:

(1) If $w=\frac{d(T x, S y)}{a}$ in (2.28), then $d(F x, G y) \preceq \frac{h}{a} d(T x, S y)$. So setting $\alpha=\frac{h}{a} \leq \frac{h}{1+\frac{2 h^{2}}{1+h}}=\frac{h^{2}+h}{2 h^{2}+h+1}=\frac{1}{2}-\frac{1-h}{2\left(2 h^{2}+h+1\right)}<\frac{1}{2}, \beta=\gamma=0$ in (2.3), it follows that $d(F x, G y) \preceq \alpha d(T x, S y)$.

(2) If $w=d(T x, F x)$ in $(2.28)$, then $d(F x, G y) \preceq h d(T x, F x)$. So setting $\beta=h<1$, $\alpha=\gamma=0, u=d(T x, F x)$ in (2.3), it follows that $d(F x, G y) \preceq \beta u$, where $u=d(T x, F x)$.

(3) If $w=d(S y, G y)$ in (2.28), then $d(F x, G y) \preceq h d(S y, G y)$. So setting $\beta=h<1$, $\alpha=\gamma=0, u=d(S y, G y)$ in (2.3), it follows that $d(F x, G y) \preceq \beta u$, where $u=d(S y, G y)$.

(4) If $w=\frac{d(T x, G y)+d(S y, F x)}{a+h}$ in $(2.28)$, then $d(F x, G y) \preceq \frac{h}{a+h}[d(T x, G y)+d(S y, F x)]$. So setting $\gamma=\frac{h}{(a+h)} \leq \frac{h}{1+\frac{2 h^{2}}{1+h}+h}=\frac{h^{2}+h}{3 h^{2}+2 h+1}=\frac{1}{3}-\frac{1-h}{3\left(3 h^{2}+2 h+1\right)}<\frac{1}{3}, \alpha=\beta=0$, $v=d(T x, G y)+d(S y, F x)$ in (2.3), it follows that $d(F x, G y) \preceq \gamma v$, where $v=d(T x, G y)+d(S y, F x)$.

Therefore, in all cases we find that $F, G, S$, and $T$ satisfy condition (2.28), then $F, G, S$, and $T$ satisfy condition (2.3).

Now, we give an example to show that condition (2.3) is more general than condition (2.28) above.

Example 2.3 Let $E=C^{1}([0,1], R), P=\{\varphi \in E: \varphi(t) \geq 0, t \in[0,1]\}, X=[0,+\infty), C=\left[\frac{3}{5}, 1\right]$, and let $d: X \times X \rightarrow E$ be defined by $d(x, y)=|x-y| \varphi$, where $\varphi \in P$ is a fixed function, e.g., 
$\varphi(t)=e^{t}$. Then $(X, d)$ is a complete cone metric space with a non-normal cone having a nonempty interior. Define $F, G, S$, and $T: C \rightarrow X$ as

$$
F(x)=\frac{2 x}{1+x}, \quad G(x)=\frac{2 x^{2}}{1+x^{2}}, \quad T(x)=x, \quad S(x)=x^{2}, \quad x \in C .
$$

Note that for all $x, y \in C$ with $x \neq y$,

$$
d(F x, G y)=\left|\frac{2 x}{1+x}-\frac{2 y^{2}}{1+y^{2}}\right| \varphi=\frac{\left|2 x-2 y^{2}\right| \varphi}{(1+x)\left(1+y^{2}\right)}=\frac{d(T x, S y)}{\frac{1}{2}(1+x)\left(1+y^{2}\right)} .
$$

Therefore, condition (2.3) is satisfied if we choose $\alpha=\frac{1}{\frac{1}{2}(1+x)\left(1+y^{2}\right)} \in\left[\frac{1}{2}, \frac{125}{144}\right], \beta=\gamma=0$.

Next, we shall see that the inequality (2.28) is not satisfied for all $0<h<1$ and $a \geq 1+\frac{2 h^{2}}{1+h}$ by taking $x=1$ and $y=\frac{3}{5}$.

Indeed, $d\left(F(1), G\left(\frac{3}{5}\right)\right)=\left|1-\frac{1}{2}\right| \varphi=\frac{1}{2} \varphi$ and $w \in\left\{\frac{d\left(T(1), S\left(\frac{3}{5}\right)\right)}{a}, d(T(1), F(1)), d\left(S\left(\frac{3}{5}\right), G\left(\frac{3}{5}\right)\right)\right.$, $\left.\frac{d\left(T(1), G\left(\frac{3}{5}\right)\right)+d\left(S\left(\frac{3}{5}\right), F(1)\right)}{a+h}\right\}=\left\{\frac{1}{a} \frac{16}{25} \varphi, 0, \frac{7}{50} \varphi, \frac{1}{a+h} \frac{57}{50} \varphi\right\}$.

Since $\frac{h}{a}<\frac{1}{2}$ and $\frac{h}{a+h}<\frac{1}{3}, d\left(F(1), G\left(\frac{3}{5}\right)\right)>h w$ for all possible cases of $w, 0<h<1$, and $a \geq 1+\frac{2 h^{2}}{1+h}$, that is, (2.3) is more general than (2.28).

So, we can obtain the following corollary.

Corollary 2.8 Let $(X, d)$ be a complete cone metric space, $C$ a nonempty closed subset of $X$ such that for each $x \in C$ and $y \notin C$ there exists a point $z \in \partial C$ such that

$$
d(x, z)+d(z, y)=d(x, y)
$$

Suppose that $F, G, S, T: C \rightarrow X$ are two pairs of non-self-mappings satisfying, for all $x, y \in C$ with $x \neq y$,

$$
d(F x, G y) \preceq h w,
$$

where $w \in\left\{\frac{d(T x, S y)}{a}, d(T x, F x), d(S y, G y), \frac{d(T x, G y)+d(S y, F x)}{a+h}\right\}$, a is a positive real number satisfying $a \geq 1+\frac{2 h^{2}}{1+h}$ and $0<h<1$. Also assume that

(I) $\partial C \subseteq S C \cap T C, F C \cap C \subseteq S C, G C \cap C \subseteq T C$,

(II) $T x \subseteq \partial C \Rightarrow F x \in C, S x \subseteq \partial C \Rightarrow G x \in C$,

(III) $S C$ and $T C$ (or FC and GC) are closed in $X$.

Then

(IV) $(F, T)$ has a point of coincidence,

(V) $(G, S)$ has a point of coincidence.

Moreover, if $(F, T)$ and $(G, S)$ are weakly compatible pairs, then $F, G, S$, and $T$ have a unique common fixed point.

The authors declare that they have no competing interests. 


\section{Author details}

'Department of Mathematics, Nanchang University, Nanchang, Jiangxi 330031, P.R. China. ${ }^{2}$ Department of Computer Science, Nanchang University, Nanchang, Jiangxi 330031, P.R. China.

\section{Acknowledgements}

The authors would like to express their sincere appreciation to the referees for their very helpful suggestions and kind comments. Project was supported by the National Natural Science Foundation of China (11361042 and 11326099) and supported partly by the Provincial Natural Science Foundation of Jiangxi, China (20142BAB201005) and the Science and Technology Project of Educational Commission of Jiangxi Province, China (GJJ11294).

Received: 7 March 2014 Accepted: 27 June 2014 Published: 22 July 2014

\section{References}

1. Huang, LG, Zhang, X: Cone metric spaces and fixed point theorems of contractive mappings. J. Math. Anal. Appl. 332(2), 1468-1476 (2007)

2. Abbas, M, Jungck, G: Common fixed point results for noncommuting mappings without continuity in cone metric spaces. J. Math. Anal. Appl. 341(1), 416-420 (2008)

3. Abbas, M, Rhoades, BE: Fixed and periodic point results in cone metric spaces. Appl. Math. Lett. 22(4), $511-515$ (2009)

4. Altun, I, Damjanović, B, Djorić, D: Fixed point and common fixed point theorems on ordered cone metric spaces. Appl. Math. Lett. 23(3), 310-316 (2010)

5. Choudhury, B, Metiya, N: Fixed points of weak contractions in cone metric spaces. Nonlinear Anal., Theory Methods Appl. 72(3-4), 1589-1593 (2010)

6. Huang, XJ, Zhu, CX, Wen, X: Common fixed point theorem for four non-self mappings in cone metric spaces. Fixed Point Theory Appl. 2010, Article ID 983802 (2010)

7. Ilić, D, Rakočević, V: Common fixed points for maps on cone metric space. J. Math. Anal. Appl. 341(2), 876-882 (2008)

8. Janković, S, Kadelburg, Z, Radenović, S: On cone metric spaces: a survey. Nonlinear Anal. 74, 2591-2601 (2011)

9. Janković, S, Kadelburg, Z, Radenović, S, Rhoades, BE: Assad-Kirk-type fixed point theorems for a pair of nonself mappings on cone metric spaces. Fixed Point Theory Appl. 2009, Article ID 761086 (2009)

10. Jungck, G, Radenović, S, Radojević, S, Rakočević, V: Common fixed point theorems for weakly compatible pairs on cone metric spaces. Fixed Point Theory Appl. 2009, Article ID 643840 (2009)

11. Kadelburg, Z, Radenović, S, Rakočević, V: A note on the equivalence of some metric and cone metric fixed point results. Appl. Math. Lett. 24, 370-374 (2011)

12. Kaekhao, A, Sintunavarat, W, Kumam, P: Common fixed point theorems of c-distance on cone metric spaces. J. Nonlinear Anal. Appl. 2012, Article ID jnaa-00137 (2012)

13. Karapinar, E, Sintunavarat, W, Kumam, P: Coupled fixed point theorems in cone metric spaces with a c-distance and applications. Fixed Point Theory Appl. 2012, 194 (2012)

14. Radenović, S: A pair of non-self mappings in cone metric spaces. Kragujev. J. Math. 36(2), 189-198 (2012)

15. Radenović, S, Rhoades, BE: Fixed point theorem for two non-self mappings in cone metric spaces. Comput. Math. Appl. 57(10), 1701-1707 (2009)

16. Rezapour, S: A review on topological properties of cone metric spaces. In: Analysis, Topology and Applications, Vrnjacka Banja, Serbia, 30 May-4 June (2008)

17. Rezapour, S, Hamlbarani, R: Some notes on the paper 'Cone metric spaces and fixed point theorems of contractive mappings'. J. Math. Anal. Appl. 345(2), 719-724 (2008)

18. Sintunavarat, W, Cho, YJ, Kumam, P: Common fixed point theorems for c-distance in ordered cone metric spaces. Comput. Math. Appl. 62, 1969-1978 (2011)

19. Sintunavarat, W, Kumam, P: Common fixed points of $f$-weak contractions in cone metric spaces. Bull. Iran. Math. Soc. 38(2), 293-303 (2012)

20. Sumitra, R, Rhymend Uthariaraj, V, Hemavathy, R, Vijayaraju, P: Common fixed point theorem for non-self mappings satisfying generalized Ćirić type contraction condition in cone metric space. Fixed Point Theory Appl. 2010, Article ID 408086 (2010)

21. Turkoglu, D, Abuloha, M: Cone metric spaces and fixed point theorems in diametrically contractive mappings. Acta Math. Sin. 26(3), 489-496 (2010)

22. Turkoglu, D, Abuloha, M, Abdeljawad, A: KKM mappings in cone metric spaces and some fixed point theorems. Nonlinear Anal., Theory Methods Appl. 72(1), 348-353 (2010)

23. Vetro, P: Common fixed points in cone metric spaces. Rend. Circ. Mat. Palermo LVI, 464-468 (2007)

24. Wardowski, D: Endpoints and fixed points of set-valued contractions in cone metric space. Nonlinear Anal., Theory Methods Appl. 71(1-2), 512-516 (2009)

25. Assad, N, Kirk, W: Fixed point theorems for set valued mappings of contractive type. Pac. J. Math. 43(3), 553-562 (1972)

26. Assad, N: On a fixed point theorem of Kannan in Banach spaces. Tamkang J. Math. 7(1), 91-94 (1976)

27. Ćirić, L: Non-self mappings satisfying non-linear contractive condition with applications. Nonlinear Anal., Theory Methods Appl. 71(7-8), 2927-2935 (2009)

28. Ćirić, L: Generalized contractions and fixed-point theorems. Publ. Inst. Math. (Belgr.) 12(26), 19-26 (1971)

29. Ćirić, L: Fixed-points for generalized multi-valued mappings. Mat. Vesn. 9(24), 265-272 (1972)

30. Ćirić, L: A remark on Rhoades fixed point theorem for non-self maps. Int. J. Math. Math. Sci. 16, 397-400 (1993)

31. Ćirić, L: On some mappings in metric spaces and fixed point theorems. Bull. Cl. Sci., Acad. R. Belg. 6(1-6), 81-89 (1995)

32. Ćirić, L: Quasi-contraction non-self mappings on Banach spaces. Bull. - Acad. Serbe Sci. Arts, Cl. Sci. Math. Nat. 23, 25-31 (1998)

33. Ćirić, L: Fixed point theorems for set-valued non-self mappings. Math. Balk. 20(2), 207-217 (2006)

34. Cirić, L: Contractive-type non-self mappings on metric spaces of hyperbolic type. J. Math. Anal. Appl. 317, 28-42 (2006)

35. Ćirić, L: Common fixed point theorems for a family of non-self mappings in convex metric spaces. Nonlinear Anal. 71(5-6), 1662-1669 (2009) 
36. Ćirić, L: Non-self mappings satisfying nonlinear contractive condition with applications. Nonlinear Anal. 71, 2927-2935 (2009)

37. Ćirić, L, Ume, J: Common fixed point theorems for multi-valued non-self mappings. Publ. Math. (Debr.) 60(3-4), 359-371 (2002)

38. Ćirić, L, Ume, J: On an extension of a theorem of Rhoades. Rev. Roum. Math. Pures Appl. 49, 103-112 (2004)

39. Ćirić, L, Cakić, N: On common fixed point theorems for non-self hybrid mappings in convex metric spaces. Appl. Math. Comput. 208(1), 90-97 (2009)

40. Ćirić, L, Ume, J, Nikolić, N: On two pairs of non-self hybrid mappings. J. Aust. Math. Soc. 83(1), 17-29 (2007)

41. Ćirić, L, Ume, J, Khan, MS, Pathak, HK: On some non-self mappings. Math. Nachr. 251, 28-33 (2003)

42. Ćirić, L, Rakočević, V, Radenović, S, Rajović, M, Lazović, R: Common fixed point theorems for non-self mappings in metric spaces of hyperbolic type. J. Comput. Appl. Math. 233, 2966-2974 (2010)

43. Kumam, P, Dung, NV, Sitthithakerngkiet, K: A generalization of Ćirić fixed point theorems. Filomat (2014, in press)

44. Hadžić, O: On coincidence points in convex metric spaces. Univ. Novom Sadu, Zb. Rad. Prir.-Mat. Fak., Ser. Mat. 19(2), 233-240 (1989)

45. Hadžić, O, Gajić, L: Coincidence points for set-valued mappings in convex metric spaces. Univ. Novom Sadu, Zb. Rad. Prir.-Mat. Fak., Ser. Mat. 16(1), 13-25 (1986)

46. Imdad, M, Kumar, S: Rhoades-type fixed-point theorems for a pair of nonself mappings. Comput. Math. Appl. 46(5-6), 919-927 (2003)

47. Rhoades, BE: A fixed point theorem for some non-self mappings. Math. Jpn. 23(4), 457-459 (1978)

48. Rhoades, BE: A fixed point theorem for non-self set-valued mappings. Int. J. Math. Math. Sci. 20(1), 9-12 (1977)

49. Ćirić, L, Ume, J: Multi-valued non-self-mappings on convex metric spaces. Nonlinear Anal., Theory Methods Appl. 60(6), 1053-1063 (2005)

50. Itoh, S: Multivalued generalized contractions and fixed point theorems. Comment. Math. Univ. Carol. 18(2), 247-258 (1977)

51. Khan, MS: Common fixed point theorems for multivalued mappings. Pac. J. Math. 95(2), 337-347 (1981)

52. Wong, YC, Ng, KF: Partially Ordered Topological Vector Spaces. Clarendon, Oxford (1973)

doi:10.1186/1687-1812-2014-157

Cite this article as: Huang et al.: Common fixed point theorem for two pairs of non-self-mappings satisfying

generalized Ćirić type contraction condition in cone metric spaces. Fixed Point Theory and Applications 2014 2014:157.

\section{Submit your manuscript to a SpringerOpen ${ }^{\circ}$ journal and benefit from:}

- Convenient online submission

Rigorous peer review

- Immediate publication on acceptance

- Open access: articles freely available online

- High visibility within the field

- Retaining the copyright to your article 